\title{
Lateral organic light-emitting diode with field-effect transistor characteristics
}

\section{AUTHOR(S):}

Oyamada, Takahito; Uchiuzou, Hiroyuki; Akiyama, Seiji; Oku, Yoshiaki; Shimoji, Noriyuki; Matsushige, Kazumi; Sasabe, Hiroyuki; Adachi, Chihaya

\section{CITATION:}

Oyamada, Takahito ... [et al]. Lateral organic light-emitting diode with field-effect transistor characteristics. JOURNAL OF APPLIED PHYSICS 2005, 98(7): 074506.

\section{ISSUE DATE:}

2005-10-01

URL:

http://hdl.handle.net/2433/39717

\section{RIGHT:}

Copyright 2005 American Institute of Physics. This article may be downloaded for personal use only. Any other use requires prior permission of the author and the American Institute of Physics. 


\title{
Lateral organic light-emitting diode with field-effect transistor characteristics
}

\author{
Takahito Oyamada and Hiroyuki Uchiuzou \\ Department of Photonics Materials Science, Chitose Institute of Science and Technology (CIST), 758-65 \\ Bibi, Chitose, Hokkaido, 066-8655, Japan
}

\author{
Seiji Akiyama \\ Optoelectronic Materials Laboratory, Research and Technology Development Division, Mitsubishi Chemical \\ Group, Science and Technology Research Center, Inc., 1000 Kamoshida, Aoba, Yokohama, 227-8502, \\ Japan \\ Yoshiaki Oku and Noriyuki Shimoji \\ New Material Device R\&D Center, Rohm Co., 21 Saiin Mizosaki, Ukyo, Kyoto, 615-8585, Japan \\ Kazumi Matsushige \\ International Innovation Center (IIC), Kyoto University, Yoshida-Honmachi, Sakyo, Kyoto, 606-8501, Japan \\ Hiroyuki Sasabe and Chihaya Adachi ${ }^{\text {a) }}$ \\ Department of Photonics Materials Science, Chitose Institute of Science and Technology (CIST), 758-65 \\ Bibi, Chitose, Hokkaido, 066-8655, Japan
}

(Received 24 May 2005; accepted 11 August 2005; published online 7 October 2005)

\begin{abstract}
We succeeded in observing bright electroluminescence (EL) from $1 \mathrm{wt} \%$-rubrene doped tetraphenylpyrene (TPPy) as an active layer in a lateral organic light-emitting diode structure that allowed field-effect transistor operation. This device configuration provides an organic light-emitting diode structure where the anode (source) and cathode (drain) electrodes are laterally arranged, providing us a chance to control the EL intensity by changing the gate bias. We demonstrated that TPPy provides compatible transistor and EL characteristics. Further, not only rubrene doping into the TPPy host but also adjusting the source-drain channel length significantly improved the EL characteristics. We observed a maximum EL quantum efficiency $\left(\eta_{\text {ext }}\right)$ of $\sim 0.5 \%$ with a $\mathrm{Cr} / \mathrm{Au}$ source $(S)$-drain $(D)$ electrode and a slightly higher $\eta_{\text {ext }}$ of $\sim 0.8 \%$ with $S$ - $D$ electrodes of $\mathrm{MgAu} / \mathrm{Au}, \mathrm{Al} / \mathrm{Au}, \mathrm{Cr} / \mathrm{YAu} / \mathrm{Au}$, and $\mathrm{MgAl} / \mathrm{Au}$ multilayers, aiming for simultaneous hole and electron injection. (C) 2005 American Institute of Physics. [DOI: 10.1063/1.2060932]
\end{abstract}

\section{INTRODUCTION}

The demand for fusing organic light-emitting diodes (OLEDs) and organic field-effect transistors (OFETs) to produce organic light-emitting FETs (OLEFETs) has been increasing and aims at achieving simplified organic active matrix displays. ${ }^{1}$ OLEFETs not only contribute to increased apertures in the pixels of light-emitting elements but also to the inexpensive fabrication of active matrix displays due to the reduced number of switching thin-film transistors (TFTs). Further, controlling the carrier accumulation and successive carrier injection from both source and drain electrodes by applying a gate bias is a unique method of providing charge carriers in organic layers. Elucidation of the detailed mechanism has attracted a great deal of attention in this research field.

Recently, there have been several reports on OLEFETs using tetracene, ${ }^{2}$ oligo-thiophene, ${ }^{3,4}$ fluorene-based polymers, ${ }^{5}$ carbon nanotubes, ${ }^{6}$ and phenylene-vinylenebased polymers. ${ }^{7}$ We also reported on OLEFETs using 2,4-bis[4-(2'-thiophene-yl)phenyl] thiophene (TPTPT) as an active layer, ${ }^{8}$ demonstrating a maximum external electroluminescence (EL) quantum efficiency of $\eta_{\mathrm{ext}}=6.3$

${ }^{a)}$ Electronic mail: c-adachi@photon.chitose.ac.jp $\times 10^{-3} \%$ with a short channel length $\left(L_{S-D}=0.8 \mu \mathrm{m}\right)$ between the source and drain $(S-D)$ electrodes. Although these reports demonstrated appreciable EL, $\eta_{\mathrm{ext}}$ is still very low, which is probably due to inefficient electron accumulation and injection from the drain electrode.

To achieve efficient OLEFETs, it has been necessary to prepare adequate organic materials with both electroluminescence and transistor characteristics. However, it is rather difficult to find candidates from well-established OLED materials, since most OLED active materials have no highperformance FET characteristics probably due to their amorphous morphologies. The recent progress in OFETs has revealed that highly packed molecular thin films with tight intermolecular $\pi$ stacking have demonstrated pronouncedly high carrier mobilities exceeding $\sim 1 \mathrm{~cm}^{2} / \mathrm{V} \mathrm{s}$. In particular, condensed aromatic compounds such as rubrene have demonstrated a high FET mobility of $\mu_{\mathrm{FET}}=\sim 10 \mathrm{~cm}^{2} / \mathrm{V} \mathrm{s}$ in the form of a single crystal. ${ }^{9}$ Instead of having a highperformance TFT function, however, most TFT active materials demonstrate rather weak photoluminescence (PL) due to their strong molecular packing, i.e., concentration quenching, meaning they are useless in light-emitting applications. Therefore, we need to satisfy the demand and find organic materials that provide both light-emitting and transistor characteristics. 


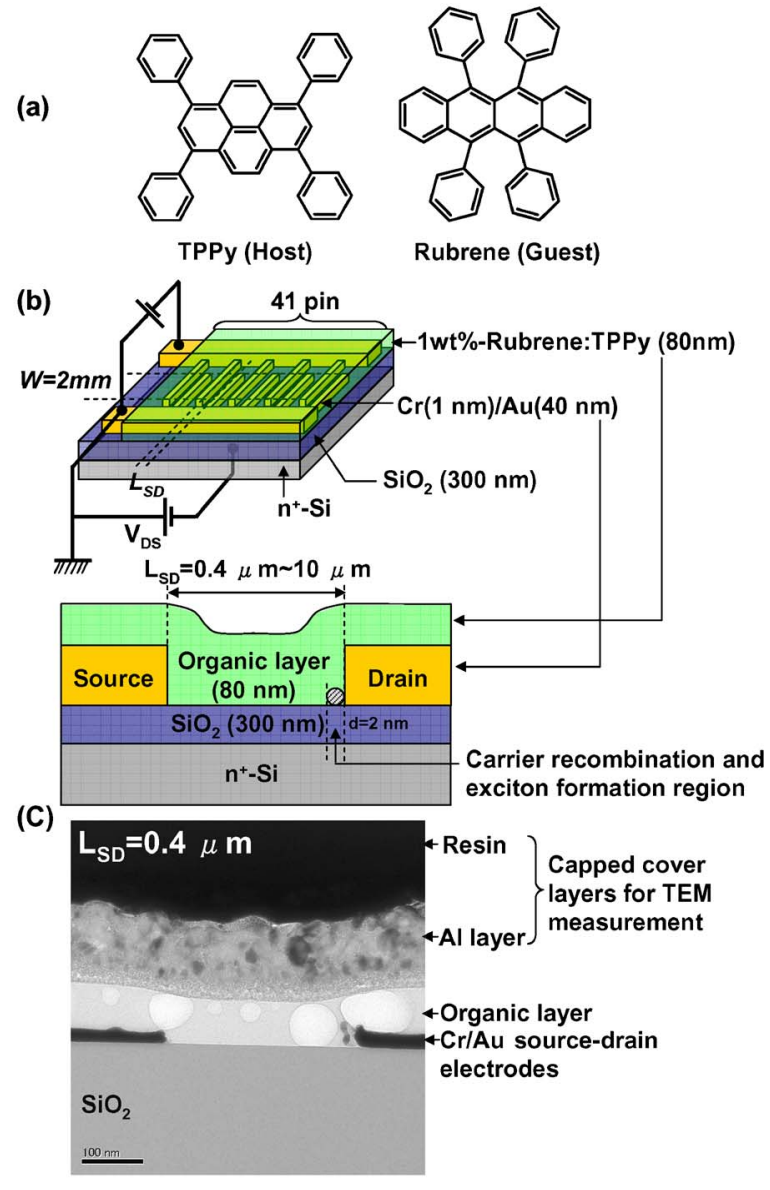

FIG. 1. (a) Molecular structures of TPPy and rubrene, and (b) schematic view of the organic light-emitting field-effect transistor (OLEFET) structure. The area of exciton formation used in calculating the light out-coupling efficiency is represented by the hatched circle. (c) Cross-sectional image of the OLEFET device structure obtained by TEM. Resin and Al layers are capped on the OLEFET device to measure the TEM image.

Since condensed aromatic polyacene derivatives generally demonstrate rather good FET characteristics and some acene derivatives, e.g., anthracene, have demonstrated high PL efficiency over $\sim 50 \%$ even in single-crystal form, we explored various polyacene derivatives and discovered that a tetraphenylpyrene (TPPy) [Fig. 1(a)] vacuum-deposited film has typical $p$-type FET characteristics with a high PL quantum efficiency of $(68 \pm 3) \%$ even in a neat vacuum-deposited film. To achieve higher PL efficiency, we doped rubrene into a TPPy host. We observed that doping various highly fluorescent molecules into a TPPy host resulted in significant improvements to PL efficiency, although some dopants completely eliminated the TFT characteristics, and adding only specific dopants effectively maintained both TFT and PL characteristics. It is likely that no significant changes to TPPy aggregated morphology, i.e., molecular packing or grain size, by rubrene doping will contribute to retaining transistor characteristics.

We also observed that the formation of a short channel length between the source and drain electrodes significantly increases carrier injection and EL efficiencies. In this study, we investigated the OLEFET characteristics of a 1 wt \%-rubrene:TPPy codeposited layer using various chan- nel lengths $\left(L_{S-D}=0.4-10 \mu \mathrm{m}\right)$, and found that the OLEFET characteristics were strongly dependent on the channel length. We also employed double-layered $\mathrm{MgAu} / \mathrm{Au}$ and $\mathrm{Al} / \mathrm{Au} S$ - $D$ electrodes, aimed at achieving both efficient hole and electron injections.

\section{EXPERIMENTAL METHOD}

Figure 1(b) is a schematic view of the fabricated OLEFET device structure. A highly doped $n^{+}$-Si (100) wafer with a 300-nm-thick $\mathrm{SiO}_{2}$ layer $\left(C_{i}=1.18 \times 10^{-8} \mathrm{~F}\right)$ was used as a gate electrode. Bottom-contact $S$ - $D$ electrodes composed of (I) $\mathrm{Cr}(0.5 \mathrm{~nm}) / \mathrm{Au}(50 \mathrm{~nm})$, (II) $\mathrm{MgAu}$ alloy ${ }^{7}$ (atom: atom ratio of 1: $1,20 \mathrm{~nm}) / \mathrm{Au}(30 \mathrm{~nm})$, (III) Al $(20 \mathrm{~nm}) / \mathrm{Au}$ $(30 \mathrm{~nm})$, (IV) $\mathrm{Cr}(1 \mathrm{~nm}) / \mathrm{YAu}$ alloy (atom: atom ratio of 1: 1, $20 \mathrm{~nm}) / \mathrm{Au}(30 \mathrm{~nm})$, and $(\mathrm{V}) \mathrm{MgAl}$ alloy (atom: atom ratio of 1: 1, $20 \mathrm{~nm}) / \mathrm{Au}(30 \mathrm{~nm})$ were formed on the $\mathrm{SiO}_{2} / n^{+}$ -Si layer using conventional photolithography and lift-off techniques. ${ }^{10}$ Here, we used symmetrical source and drain electrode configuration where both the electrodes are made of the same materials. The channel lengths between the $S$ - $D$ electrodes were $L_{S-D}=0.4,0.6,0.8,1,3$, and $10 \mu \mathrm{m}$, with a channel width of $2 \mathrm{~mm}$. After the photolithography process, the substrate was degreased with acetone and isopropanol and cleaned before being loaded into an evaporation system. An 80-nm-thick 1 wt \%-rubrene-doped TPPy film as an active layer was deposited onto the $S-D$ electrodes in a vacuum of $<1 \times 10^{-3} \mathrm{~Pa}$. The deposition rates for rubrene and TPPy were maintained at 0.005 and $0.5 \mathrm{~nm} / \mathrm{s}$, respectively. Figure $1(\mathrm{c})$ is a transmission electron microscopy (TEM) image of an OLEFET device with $L_{S-D}=0.4 \mu \mathrm{m}$. The source and drain electrodes are well fabricated with almost an ideal structure, while some imperfections in the source and drain shapes at the edges can be observed that were induced during the lift-off process, and which could not be controlled during the photolithography process.

All measurements were carried out in a vacuum of $\sim 1$ $\times 10^{-3} \mathrm{~Pa}$ at room temperature, since the device demonstrated unstable TFT characteristics in air. The dependence of the drain current $\left(I_{d}\right)$-drain voltage $\left(V_{d}\right)$-luminance $(L)$ characteristics on the gate voltage $\left(V_{g}\right)$ were measured with an Agilent 4145 semiconductor parameter analyzer with the quantum efficiency directly obtained by centering a highly sensitive photon counter (Hamamatsu, C767) and a calibrated $\mathrm{Si}$ photodiode. The $V_{d}$ was scanned from 10 to $-100 \mathrm{~V}$ in $-1 \mathrm{~V}$ steps and the $V_{g}$ was scanned from 0 to $-100 \mathrm{~V}$ in $-20 \mathrm{~V}$ steps. EL spectra were measured with a multichannel spectrometer (Hamamatsu, PMA-11).

The absorption spectra and PL quantum efficiency of the organic layers were measured with a fluorospectrometer (FP6300, Jasco) and an UV/VIS spectrometer (UV-3100, Shimadzu), and an absolute PL quantum efficiency measurement system using an integrating sphere. ${ }^{11}$ Also, the work function (WF) and the highest occupied molecular-orbital (HOMO) level of the metal and the organic layers were measured using ultraviolet photoelectron spectroscopy, AC-1 (Riken Keiki Co.). 


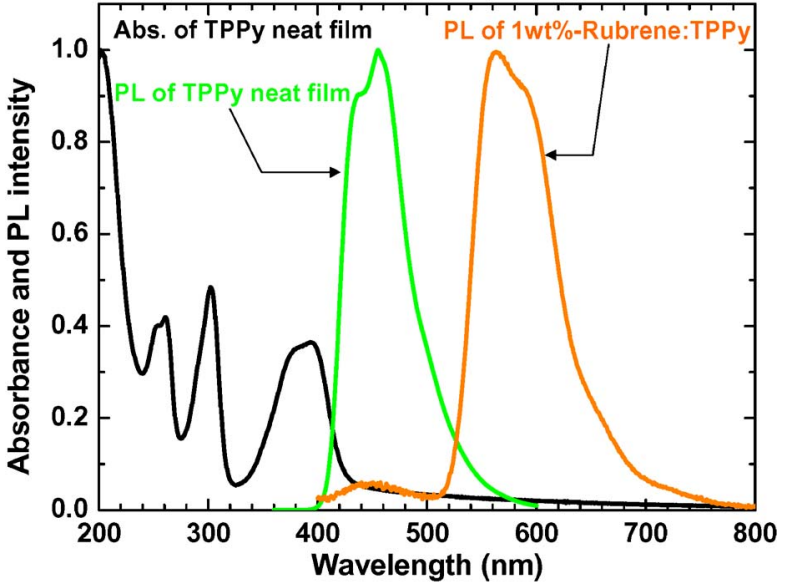

FIG. 2. Photoluminescence (PL) and absorption spectra for TPPy and $1 \mathrm{wt} \%$-rubrene:TPPy codeposited layers. The rubrene:TPPy film showed PL efficiency of $\phi_{\mathrm{PL}}=(99 \pm 1) \%$, demonstrating complete energy transfer from TPPy into rubrene and radiative decay from rubrene's singlet excited state.

\section{RESULTS AND DISCUSSION}

Figure 2 plots the PL spectra for a 1 wt \%-rubrene:TPPy codeposited film $(100 \mathrm{~nm})$ and a TPPy neat film $(100 \mathrm{~nm})$ with the absorption spectrum for the TPPy neat layer. Although the TPPy neat film exhibited a PL peak at $455 \mathrm{~nm}$, the 1 wt \%-rubrene:TPPy film had strong PL with an emission peak at $\lambda_{\max }=559 \mathrm{~nm}$ that was based on rubrene, suggesting efficient Förster energy transfer from TPPy to rubrene occurred. Here, the $1 \mathrm{wt} \%$-rubrene-doped TPPy codeposited layer demonstrated an ultimate PL efficiency of $\phi_{\mathrm{PL}}=(99 \pm 1) \%$, while the TPPy film demonstrated $\Phi_{\mathrm{PL}}$ $=(68 \pm 3) \%$.

Figure 3 plots $I_{d^{-}} V_{d}$ characteristics with various channel lengths. The OLEFET with $L_{S-D}=10 \mu \mathrm{m}$ (a) exhibited typical unipolar FET characteristics with linear behavior in the low $V_{d}$ region $(\varsigma-40 \mathrm{~V})$ and typical saturation behavior in the high $V_{d}$ region $(\geq-50 \mathrm{~V})$, indicating a depletion region had formed near the drain electrode under saturation conditions $\left(V_{d} \geq-50 \mathrm{~V}\right)$. The OLEFET with a short channel length of $L_{S-D} \lesssim 1 \mu \mathrm{m}$, on the other hand, demonstrated unusual $I_{d^{-}} V_{d}$ characteristics. The $I_{d}$ progressively increased and divergent behavior in $I_{d^{-}} V_{d}$ characteristics was observed. In particular, a very high current of $I_{d}$ was observed in device (f) even with $V_{g}=0 \mathrm{~V}$.

Figure 4 summarizes the external EL quantum efficiency $\left(\eta_{\text {ext }}\right)$-drain current $\left(I_{d}\right)$ characteristics depending on various channel lengths. The $\eta_{\text {ext }}$ of OLEFETs with long channel lengths of $L_{S-D}=10$ and $3 \mu \mathrm{m}$ demonstrated very rapid increases in $\eta_{\text {ext }}$ in the drain current compared with that of other devices. The onset of light emission roughly corresponds to the onset of saturation current, suggesting that efficient electron injection begins at the onset of the saturation current region, corresponding to the formation of a depletion region. Since $1 \mathrm{wt} \%$-rubrene:TPPy had typical $p$-type FET characteristics, the major accumulated carriers in the $1 \mathrm{wt} \%$-rubrene:TPPy layer are holes and the minority carrier injection of electrons is enhanced with increasing $V_{d}$. The OLEFET with a short channel length $\left(L_{S-D} \lesssim 1 \mu \mathrm{m}\right)$, on the
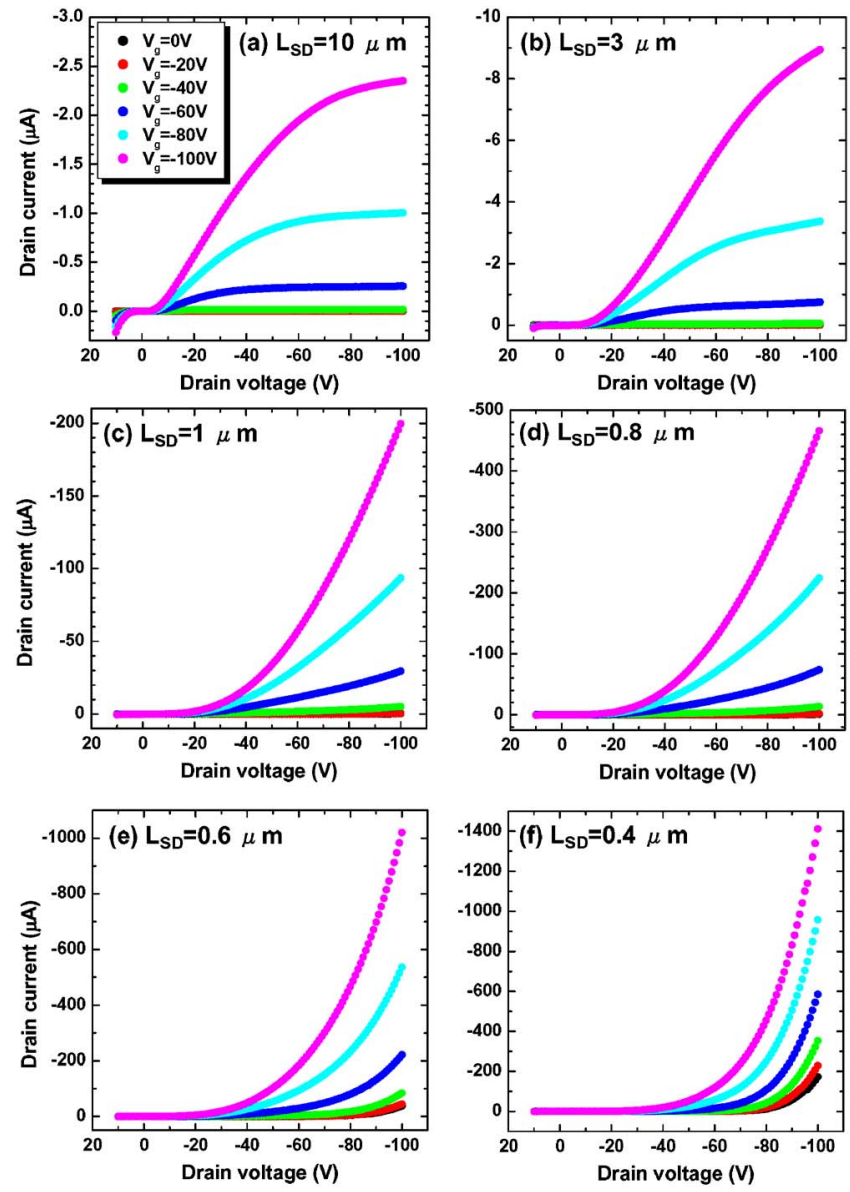

FIG. 3. Drain current $\left(I_{d}\right)$ vs drain voltage $\left(V_{d}\right)$ characteristics depending on channel length between source and drain electrodes: (a) $L_{S-D}=10 \mu \mathrm{m}$, (b) $3 \mu \mathrm{m}$, (c) $1 \mu \mathrm{m}$, (d) $0.8 \mu \mathrm{m}$, (e) $0.6 \mu \mathrm{m}$, and (f) $0.4 \mu \mathrm{m}$ in OLEFETs with $1 \mathrm{wt} \%$-rubrene:TPPy layer as active layer. Various gate voltages $\left(V_{g}=0\right.$, $-20,-40,-60,-80$, and $-100 \mathrm{~V}$ ) were applied to these devices. Although the devices with long channel lengths of $L_{S-D}=10$ and $3 \mu \mathrm{m}$ showed typical $p$-type transistor characteristics, the devices with short channel lengths of $L_{S-D}<1 \mu \mathrm{m}$ showed a rapid increase of $I_{d}$ with an increase of $V_{d}$.

other hand, demonstrated a rather gradual increase in $\eta_{\text {ext }}$ with an increase in $I_{d}$, indicating that the operation mechanism was significantly different from those with long channel lengths of $L_{S-D}=10$ and $3 \mu \mathrm{m}$. The formation of the short $S$ - $D$ channel enhanced electron injection efficiency from the drain electrode even in the low $V_{d}$ region, leading to enhanced $\eta_{\text {ext }}$. In particular, the OLEFET with a channel length of $L_{S-D}=1.0 \mu \mathrm{m}$ demonstrated the highest $\eta_{\text {ext }}$ of $0.47 \%$.

These $I_{d^{-}} V_{d}$ and $\eta_{\text {ext }} I_{d}$ characteristics meant that OLEFETs with longer channel lengths of $L_{S-D}=10$ and $3 \mu \mathrm{m}$ and a shorter channel length of $L_{S-D} \lesssim 1 \mu \mathrm{m}$ demonstrated quite different FET characteristics. Figure 5 summarizes $I_{d}-V_{d}$ and $\eta_{\text {ext }}-I_{d}$ characteristics and their potential alignment in OLEFETs with $L_{S-D}=0.4 \mu \mathrm{m}$ under the application of various positive and negative gate biases and drain voltages: (a) $V_{g}(-)$ and $V_{D}(-)$, (b) $V_{g}(+)$ and $V_{D}(-)$, and (c) $V_{g}(+)$ and $V_{D}(+)$. Although OLEFETs with longer channel lengths had typical $p$-type transistor characteristics, in those with short channel lengths, direct carrier injection from the source and drain electrodes, due to the high electrical field between them, mainly controlled the $I_{d^{-}}-V_{d}$ characteristics [Fig. 5(a)]. The OLEFET with $L_{S-D}=0.4 \mu \mathrm{m}$ had well-balanced hole and 

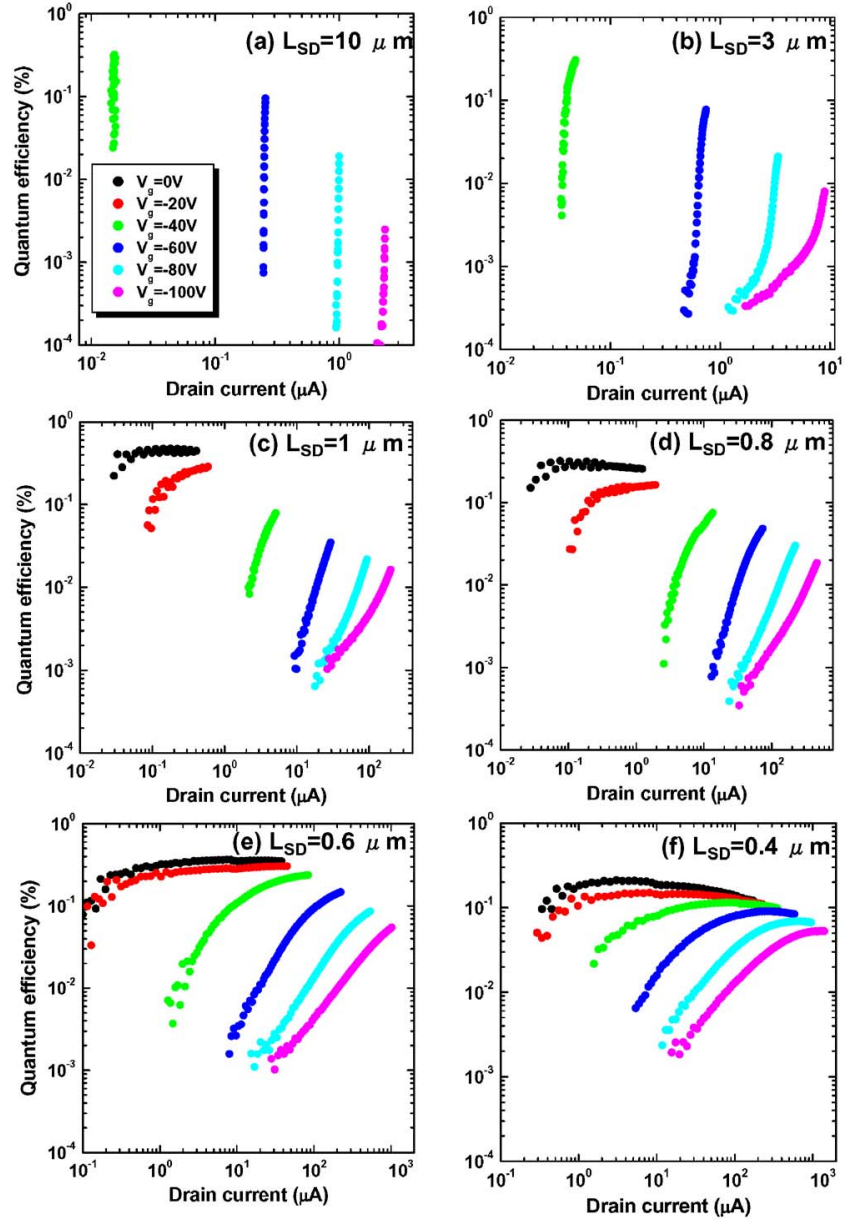

FIG. 4. External electroluminescence (EL) quantum efficiency $\left(\eta_{\text {ext }}\right)$ vs drain voltage $\left(V_{d}\right)$ characteristics depending on channel length between source and drain electrodes; (a) $L_{S-D}=10 \mu \mathrm{m}$, (b) $3 \mu \mathrm{m}$, (c) $1 \mu \mathrm{m}$, (d) $0.8 \mu \mathrm{m}$, (e) $0.6 \mu \mathrm{m}$, and (f) $0.4 \mu \mathrm{m}$ in OLEFETs with $1 \mathrm{wt} \%$-rubrene:TPPy layer as active layer. Various gate voltages $\left(V_{g}=0\right.$, $-20,-40,-60,-80$, and $-100 \mathrm{~V})$ were applied to these devices.

electron injection when $V_{g}=0 \mathrm{~V}$, which can be proved by almost the complete lack of roll-off characteristics of $\eta_{\text {ext }}$ on $I_{d}$. With an increase in the negative voltage of $V_{g}$, on the other hand, $\eta_{\text {ext }}$ gradually increased with $I_{d}$, suggesting that inferior electron injection compared with hole injection occurs in the low $V_{S-D}$ region, and the ratio of hole and electron densities changes depending on $V_{S-D}$.

From the $\eta_{\text {ext }}-V_{d^{-}} V_{g}$ characteristics of all OLEFET devices, we recognized that an increase in negative gate voltage significantly decreased $\eta_{\text {ext }}$, although $I_{d}$ dramatically increased in all devices. This suggests that applying a negative gate voltage enhances $I_{d}$ due to the accumulation of holes at the $\mathrm{SiO}_{2}$ /organic interface and electron accumulation and injection are weakened. Therefore, to enhance electron injection and accumulation, we need to apply a positive gate voltage. Figure 5(b) plots $I_{d^{-}} V_{d}$ and $\eta_{\text {ext }} I_{d}$ characteristics with a positive $V_{g}$ and negative $V_{d}$ arrangement. Here, we can expect large electron accumulation due to the large difference in negative potential between $V_{d}$ and $V_{g}$ with the suppression of hole accumulation. However, we observed no $I_{d}$ modulation, suggesting that no net electron accumulation occurs even with a large positive voltage of $V_{g}$ and $I_{d}$ is simply

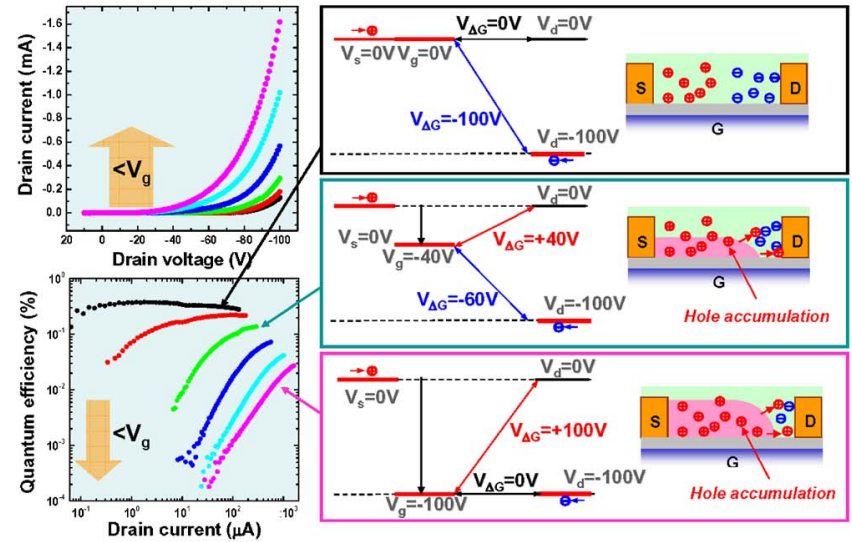

(a)
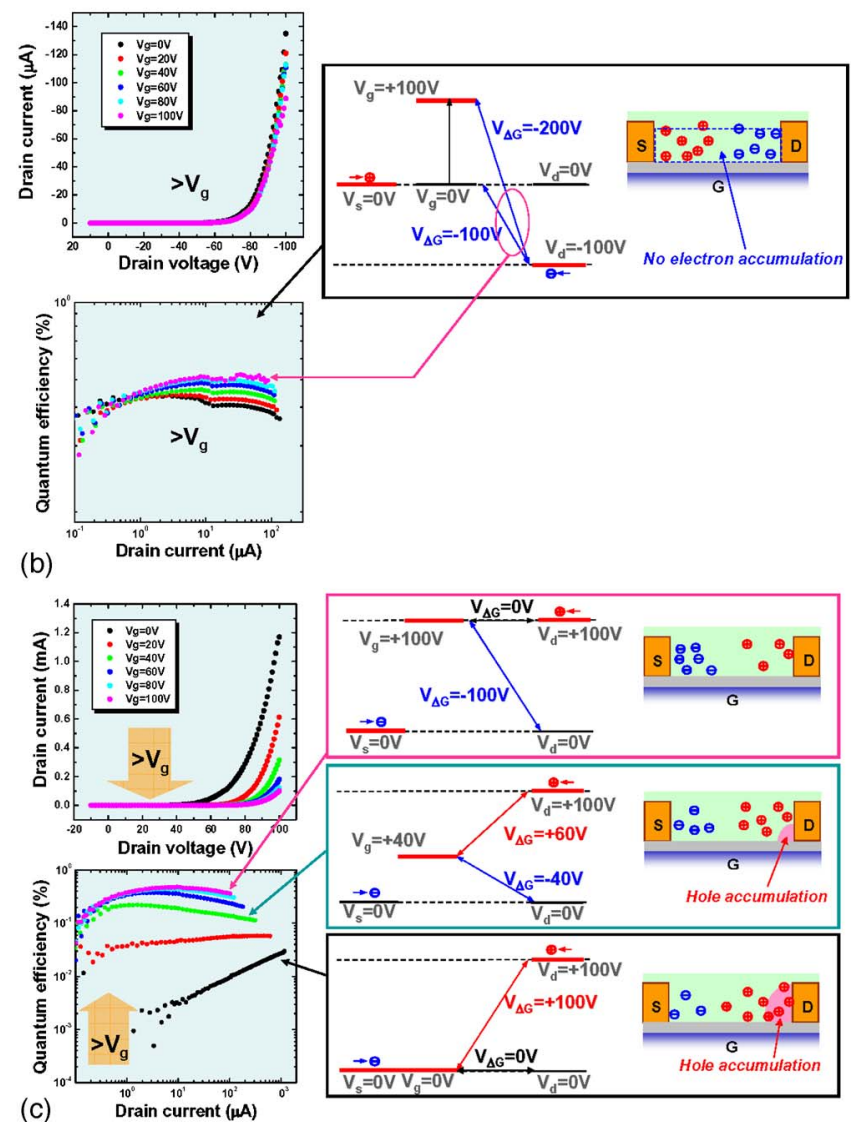

FIG. 5. Drain current $\left(I_{d}\right)$ vs drain voltage $\left(V_{d}\right)$ and external EL quantum efficiency $\left(\eta_{\text {ext }}\right)$ vs drain current $\left(I_{d}\right)$ characteristics depending on gate voltage $\left(V_{g}\right)$ in OLEFETs with $L_{S-D}=0.4 \mu \mathrm{m}$ : (a) $V_{g}<0, V_{d}<0$, (b) $V_{g}>0$, $V_{d}<0$, and (c) $V_{g}>0, V_{d}>0$. The potential diagram and schematic view of carrier accumulation are shown. (a) With increased negative gate voltage $\left(V_{g}\right)$, hole accumulation occurs and unbalanced hole and electron accumulation decreases $\eta_{\text {ext }}$. Highest $\eta_{\text {ext }}$ and balanced hole and electron injection was observed with $V_{g}=0 \mathrm{~V}$, suggesting that no net electron accumulation occurs in the rubrene:TPPy layer. (b) Even with high positive $V_{g}$, which can be expected to enhance electron accumulation, no amplification of $I_{d}$ was observed, suggesting no net electron accumulation. (c) $I_{d}-V_{d}$ and $\eta_{\text {ext }}-I_{d}$ characteristics are similar to those in device (a).

controlled by direct injection of holes and electrons from both the source and drain electrodes, respectively. A slight increase in $\eta_{\text {ext }}$ with higher $V_{g}$ indicates slightly enhanced electron injection occurs from the drain electrode with higher $V_{g}$. Further, with positive $V_{g}$ and $V_{d}\left[\right.$ Fig. 5(c)], the $I_{d^{-}} V_{d}$ and $\eta_{\text {ext }} I_{d}$ characteristics were similar to that in (Fig. 5(a) since 
(a)

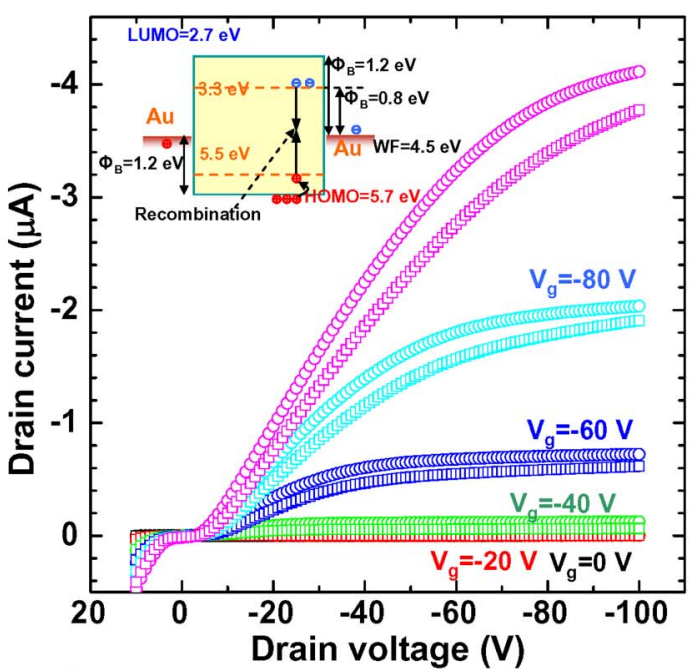

(b)

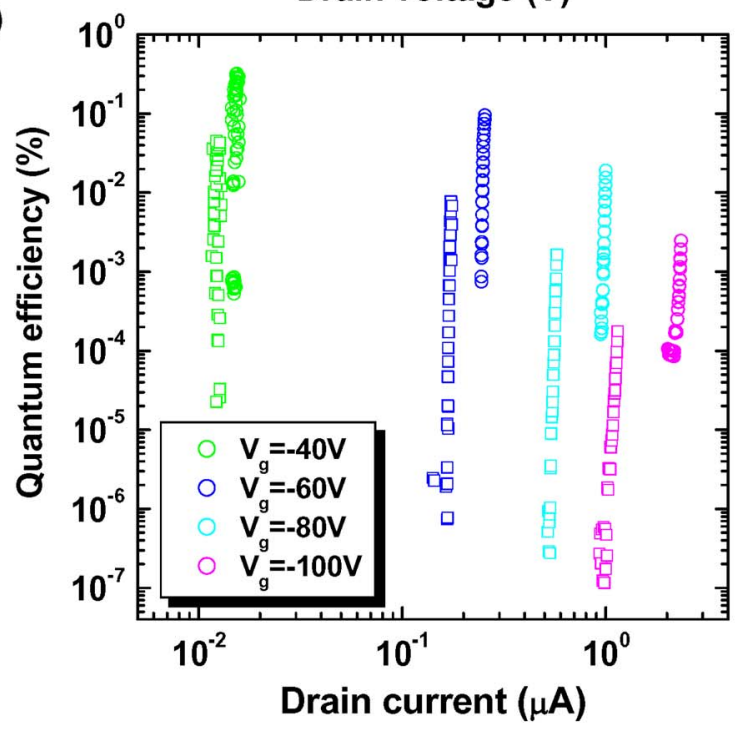

FIG. 6. (a) Drain current $\left(I_{d}\right)$ vs drain voltage $\left(V_{d}\right)$ and (b) external EL quantum efficiency $\left(\eta_{\text {ext }}\right)$ vs drain current $\left(I_{d}\right)$ characteristics of $1 \mathrm{wt} \%$-rubene:TPPy (circles) and TPPy layers (squares) in OLEFETs with $\mathrm{Cr} / \mathrm{Au} S-D$ electrodes $\left(L_{S-D}=10 \mu \mathrm{m}\right)$. (Inset) Schematic view of the energylevel diagram for $S-D$ drain electrode and organic layer in OLEFETs.

the potential difference among the three electrodes was basically the same in (a) and (c) devices. An ideal situation where there is potential distribution in the light- emitting transistors should induce both hole and electron accumulations simultaneously in the organic active layer. However, our FET characteristics indicated no net electron accumulation even with large $V_{\mathrm{DG}}=V_{g}-V_{d}=200 \mathrm{~V}$, while hole accumulation was easily accomplished. This was probably due to the intrinsic characteristics of $p$-type accumulation in the TPPy layer. To enhance $\eta_{\text {ext }}$, we have to introduce active materials that are capable of both hole and electron accumulation.

Let us now compare FET characteristics between 1 wt \%-rubrene:TPPy and TPPy layers (Fig. 6). The $I_{d}-V_{d}$ curves had very similar characteristics with almost the same FET mobilities of $\mu_{\mathrm{FET}}=1.7 \times 10^{-5} \mathrm{~cm}^{2} / \mathrm{V} \mathrm{s}$. The 1 wt \%-rubrene:TPPy, on the other hand, had ten times higher $\eta_{\text {ext }}$ than that of a TPPy layer, demonstrating that rubrene doping greatly enhances electron injection effi-
TABLE I. The OLEFET characteristics: the maximum $I_{d}, \eta_{\mathrm{ext}}$, and $L_{\mathrm{max}}$ depending on the channel length and $S$-D electrodes; (I) $\mathrm{Cr}(1 \mathrm{~nm}) / \mathrm{Au}$ (40 nm), (II) $\mathrm{MgAu}$ alloy $(20 \mathrm{~nm}) / \mathrm{Au}(30 \mathrm{~nm})$, (III) Al $(20 \mathrm{~nm}) / \mathrm{Au}$ $(30 \mathrm{~nm})$, (IV) $\mathrm{Cr}(1 \mathrm{~nm}) / \mathrm{YAu}$ alloy $(20 \mathrm{~nm}) / \mathrm{Au}(30 \mathrm{~nm})$, and (V) $\mathrm{MgAl}$ alloy $(20 \mathrm{~nm}) / \mathrm{Au}(30 \mathrm{~nm})$ double-layer electrodes. $\left(E_{\max }\right.$ : electrical field between $S$ - $D$ electrodes at $V_{d}=-100 \mathrm{~V}$.)

\begin{tabular}{|c|c|c|c|c|}
\hline$L_{S-D}(\mu \mathrm{m})$ & $E_{\max }(\mathrm{MV} / \mathrm{cm})$ & Max. $I_{d}(\mu \mathrm{A})$ & Max. $\eta_{\mathrm{ext}}(\%)$ & $L_{\max }\left(\mathrm{cd} / \mathrm{m}^{2}\right)$ \\
\hline \multicolumn{5}{|c|}{$S$ - $D$ electrode: $\mathrm{Cr}(1 \mathrm{~nm}) / \mathrm{Au}(40 \mathrm{~nm})$} \\
\hline 10 & 0.10 & -4.1 & 0.29 & 0.48 \\
\hline 3 & 0.33 & -22 & 0.30 & 1.3 \\
\hline 1 & 1.0 & -200 & 0.47 & 10 \\
\hline 0.8 & 1.3 & -285 & 0.36 & 22 \\
\hline 0.6 & 1.7 & -1020 & 0.37 & 150 \\
\hline 0.4 & 2.5 & -1410 & 0.22 & 193 \\
\hline \multicolumn{5}{|c|}{$S-D$ electrode: $\mathrm{MgAu}(20 \mathrm{~nm}) / \mathrm{Au}(30 \mathrm{~nm})$} \\
\hline 10 & 0.10 & -0.089 & 0.30 & 0.004 \\
\hline 3 & 0.33 & -0.19 & 0.42 & 0.024 \\
\hline 1 & 1.0 & -4.3 & 0.75 & 11 \\
\hline 0.8 & 1.3 & -68 & 0.71 & 65 \\
\hline 0.6 & 1.7 & -262 & 0.70 & 194 \\
\hline 0.4 & 2.5 & -2250 & 0.43 & 643 \\
\hline \multicolumn{5}{|c|}{$S-D$ electrode: $\mathrm{Al}(20 \mathrm{~nm}) / \mathrm{Au}(30 \mathrm{~nm})$} \\
\hline 10 & 0.10 & -1.8 & 0.13 & 0.14 \\
\hline 3 & 0.33 & -26 & 0.06 & 0.38 \\
\hline 1 & 1.0 & -390 & 0.60 & 34 \\
\hline 0.8 & 1.3 & -757 & 0.56 & 87 \\
\hline 0.6 & 1.7 & -920 & 0.56 & 108 \\
\hline 0.4 & 2.5 & -1720 & 0.16 & 160 \\
\hline \multicolumn{5}{|c|}{$S$ - $D$ electrode: $\mathrm{Cr}(1 \mathrm{~nm}) / \mathrm{YAu}(20 \mathrm{~nm}) / \mathrm{Au}(30 \mathrm{~nm})$} \\
\hline 10 & 0.10 & -0.67 & 0.10 & 0.00034 \\
\hline 3 & 0.33 & -2.8 & 0.11 & 0.0061 \\
\hline 1 & 1.0 & -114 & 0.66 & 1.6 \\
\hline 0.8 & 1.3 & -465 & 0.46 & 0.94 \\
\hline 0.6 & 1.7 & -747 & 0.17 & 13 \\
\hline 0.4 & 2.5 & -868 & 0.11 & 16 \\
\hline \multicolumn{5}{|c|}{$S-D$ electrode: $\mathrm{MgAl}(20 \mathrm{~nm}) / \mathrm{Au}(30 \mathrm{~nm})$} \\
\hline 10 & 0.10 & -0.56 & 0.16 & 0.0041 \\
\hline 3 & 0.33 & -3.6 & 0.19 & 0.031 \\
\hline 1 & 1.0 & -43 & 0.79 & 0.97 \\
\hline 0.8 & 1.3 & -71 & 0.66 & 6.2 \\
\hline 0.6 & 1.7 & -297 & 0.48 & 86 \\
\hline 0.4 & 2.5 & -6250 & 0.15 & 273 \\
\hline
\end{tabular}

ciency. Since the lowest unoccupied molecular orbital (LUMO) level of rubrene appears at $3.3 \mathrm{eV}$, which is $0.6 \mathrm{eV}$ deeper than that of a TPPy layer $(2.7 \mathrm{eV})$, electrons are efficiently injected from the Au drain electrode into the LUMO level of rubrene.

Table I summarizes the OLEFET characteristics for maximum $I_{d}, \eta_{\mathrm{ext}}$, and maximum luminance $\left(L_{\max }\right)$ depending on the channel length with various $S-D$ metals: (I) $\mathrm{Cr} / \mathrm{Au}$, (II) $\mathrm{MgAu} / \mathrm{Au}$, (III) $\mathrm{Al} / \mathrm{Au}$, and (IV) $\mathrm{Cr} / \mathrm{YAu} / \mathrm{Au}$ electrodes and $(\mathrm{V}) \mathrm{MgAl} / \mathrm{Au}$ double-layer electrodes. Figure 7 plots the maximum $\eta_{\text {ext }}$ depending on the $S-D$ electrode materials. These double-layered $S-D$ electrodes with low-WF metals (Au: $\sim 4.5 \mathrm{eV}, \mathrm{MgAu}$ alloy: $\sim 3.8 \mathrm{eV}, \mathrm{Al}: \sim 4.2 \mathrm{eV}$, YAu alloy: $\sim 4.1 \mathrm{eV}$, and $\mathrm{MgAl}$ alloy: $\sim 3.4 \mathrm{eV}$ ) had a maximum $\eta_{\text {ext }}$ of $\sim 0.75 \%, \sim 0.60 \%, \sim 0.66 \%$, and $\sim 0.79 \%$, re- 


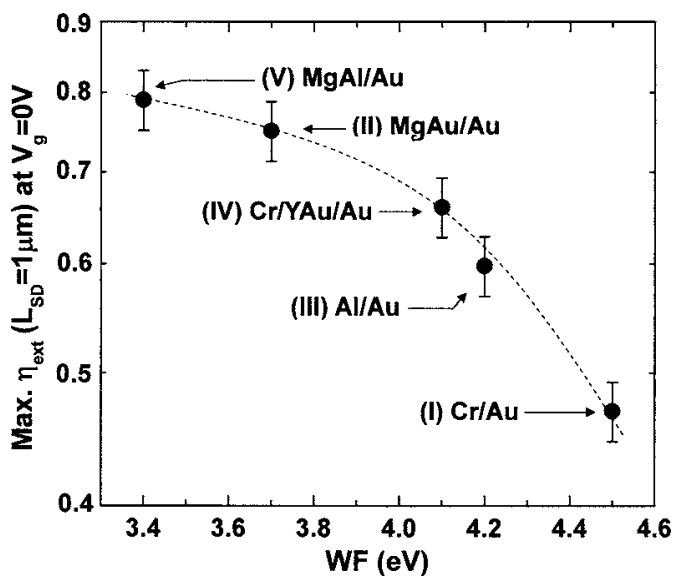

FIG. 7. Maximum external EL quantum efficiency $\left(\eta_{\text {ext }}\right)$ at $V_{g}=0 \mathrm{~V}$ vs work-function (WF) characteristics depending on $S-D$ electrode materials in OLEFETs with $L_{S-D}=1 \mu \mathrm{m}$.

spectively, which was $\sim 1.5$ times higher than that of the device with a $\mathrm{Cr} / \mathrm{Au}$ electrode, indicating that the decreased electron injection barrier by the low-WF materials enhanced $\eta_{\mathrm{ext}}$.

Here, we will estimate the carrier-injection balance factor $(\gamma)$, i.e., the hole/electron ratio, to examine the ratedetermining process for EL efficiency. We used a simulation program of LIGHTTOOLS (see Ref. 11) to estimate the light out-coupling efficiency $\left(\eta_{p}\right)$ in the OFET structure, as shown in Fig. 1(b) [(refractive index: $n$, reflectivity index: $r$, and transmittance: $t$ ); active layer ( $1 \mathrm{wt} \%$-rubrene:TPPy film): $n \approx 1.79$ and $t=100 \%$ at about $\lambda=560 \mathrm{~nm}, S-D$ electrodes $(\mathrm{Au}): \quad r \approx 80 \%$, insulator $\left(\mathrm{SiO}_{2}\right.$ by thermal-oxidation method): $n \approx 1.46$ and $t=100 \%$ at $\lambda=350-800 \mathrm{~nm}$, and gate electrode $\left(n^{+}-\mathrm{Si}\right): n \approx 3.6$ and $r \approx 30 \%$ at $\lambda=400-800 \mathrm{~nm}$ ]. The multiple interference effect was not considered for this estimate. We obtained maximum $\eta_{\text {ext }}$ of $\sim 0.47 \%, \sim 0.75 \%$, $\sim 0.60 \%, \sim 0.66 \%$, and $\sim 0.79 \%$ and the maximum $\gamma$ of short channel devices $\left(L_{S-D}=1 \mu \mathrm{m}\right)$ with $\mathrm{Cr} / \mathrm{Au}, \mathrm{MgAu} / \mathrm{Au}$, $\mathrm{Al} / \mathrm{Au}, \mathrm{Cr} / \mathrm{YAu} / \mathrm{Au}$, and the $\mathrm{MgAl} / \mathrm{Au}$ double-layer $S-D$ electrode reached $\gamma_{\max } \sim 30 \%, \gamma_{\max } \sim 50 \%, \gamma_{\max } \sim 40 \%$, $\gamma_{\max } \sim 44 \%$, and $\gamma_{\max } \sim 53 \%$, respectively. Thus, the electron injection efficiency is still a rate-limiting process for obtaining higher $\eta_{\text {ext }}$, although electron injection efficiency was increased by using the low-WF $S$ - $D$ electrodes.

Figure 8 plots (a) EL spectra depending on the gate voltage and (b) is a photograph of light emission in an OLEFET with a $\mathrm{Cr} / \mathrm{Au} S-D$ electrode and a short channel length of $L_{S-D}=0.6 \mu \mathrm{m}$ under $V_{d}=-100 \mathrm{~V}$. The device exhibited characteristic rubrene emission and EL intensity progressively increased with increasing $V_{g}$. The light- emission image of the OLEFET shows luminance of $\sim 150 \mathrm{~cd} / \mathrm{m}^{2}$ at $V_{d}=$ $-100 \mathrm{~V}$ and $V_{g}=-100 \mathrm{~V}$. The EL intensity was able to be controlled well by changing the gate voltage. The EL spectra exhibited a maximum emission peak of $560 \mathrm{~nm}$ and a shoulder peak at $600 \mathrm{~nm}$, which coincided well with the PL of the codeposited film (Fig. 2).

In summary, we demonstrated bright EL from an OFET structure with a $1 \mathrm{wt} \%$-rubrene:TPPy codeposited film as an active layer. Our device configuration provided a lightemitting diode structure where the anode and cathode elec-

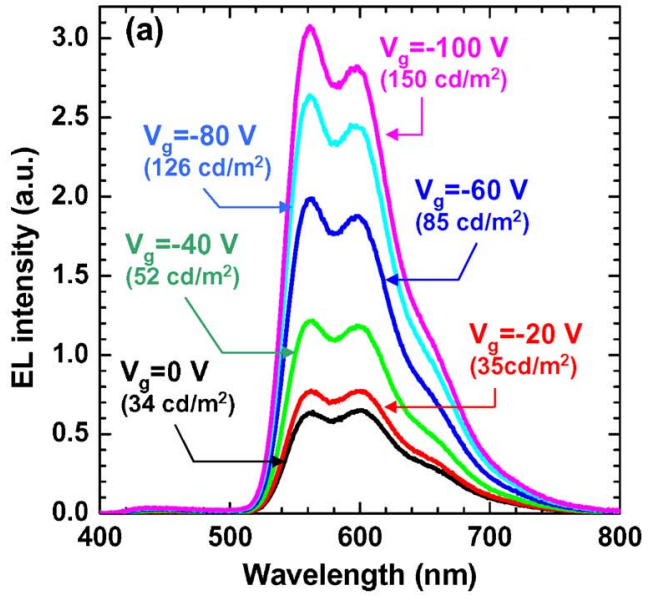

(b)

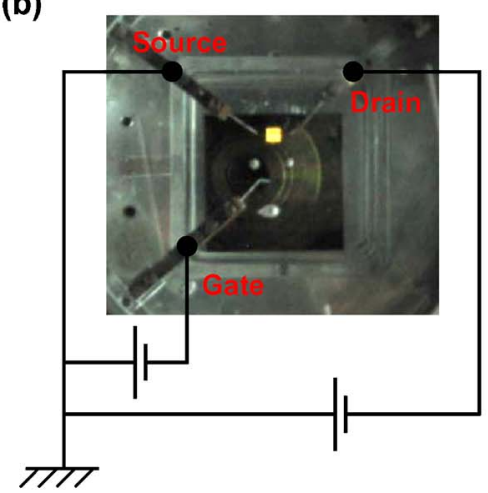

FIG. 8. (a) EL spectra depending on gate voltage $\left(V_{g}\right)$ in OLEFETs with 1 wt \%-rubene:TPPy layer and $\mathrm{Cr} / \mathrm{Au} S$ - $D$ electrodes, and (b) a photograph of light-emission OLEFETs with a short channel length of $L_{S-D}=0.6 \mu \mathrm{m}$.

trodes were laterally arranged, which enabled us to control the EL intensity by changing the gate bias. We obtained a maximum $\eta_{\text {ext }}$ of $\sim 0.8 \%$ in a short channel device with $\mathrm{MgAl} / \mathrm{Au}$ double-layer $S-D$ electrodes, demonstrating significant improvements in electron injection and EL efficiency using a short channel length of $L_{S-D} \lesssim 1 \mu \mathrm{m}$. We clarified that electron accumulation and injection still limit device performance.

\section{ACKNOWLEDGMENT}

This work was supported by the Integrated Industry Academia Partnership (IIAP) of the Kyoto University International Innovation Center.

${ }^{1}$ R. M. A. Dawson et al., SID Int. Symp. Digest Tech. Papers 29, 11 (1998).

${ }^{2}$ C. Rost, S. F. Karg, W. Riess, M. A. Loi, M. Murgia, and M. Muccini, Appl. Phys. Lett. 85, 1613 (2004).

${ }^{3}$ A. Hepp, H. Heil, W. Weise, M. Ahles, R. Schmechel, and H. von Seggern, Phys. Rev. Lett. 91, 157406 (2003).

${ }^{4}$ C. Rost, S. F. Karg, W. Riess, M. A. Loi, M. Murgia, and M. Muccini, Appl. Phys. Lett. 85, 1613 (2004).

${ }^{5}$ M. Ahles, A. Hepp, R. Schmechel, and H. von Seggern, Appl. Phys. Lett. 84, 428 (2004).

${ }^{6}$ J. A. Misewich, R. Martel, Ph. Avouris, J. C. Tsang, S. Heinze, and J. Tersoff, Science 300, 783 (2003).

${ }^{7}$ T. Sakanoue, E. Fujiwara, R. Yamada, and H. Tada, Appl. Phys. Lett. 84, 3037 (2004). 
${ }^{8}$ T. Oyamada, S. Okuyama, N. Shimoji, K. Matsushige, H. Sasabe, and C. Adachi, Appl. Phys. Lett. 86, 093505 (2005).

V. C. Sundar, J. Zaumseil, V. Podzorov, E. Menard, R. L. Willett, T. Someya, M. E. Gershenson, and J. A. Rogers, Science 303, 1644 (2004).
${ }^{10}$ T. Oyamada, H. Sasabe, and C. Adachi, IEEJ Transactions on Electronics, Information and Systems 124C, 1216 (2004).

${ }^{11}$ Y. Kawamura, H. Sasabe, and C. Adachi, Jpn. J. Appl. Phys., Part 1 43, 7729 (2004). 